DOI: https://doi.org/10.46296/rc.v2i4.0009

\title{
Postventa y desarrollo comercial en las PYMES
}

\section{After-sales and commercial development in SMEs}

\author{
Autor: Chiriboga-Mendoza Fidel Ricardo \\ Universidad Laica Eloy Alfaro de Manabí (ULEAM). Manta, Ecuador. \\ fchiriboga35@gmail.com \\ Autor: Zambrano-Pilay Enrique Cristóbal \\ Universidad Laica Eloy Alfaro de Manabí (ULEAM). Manta, Ecuador. \\ eczp_1997@hotmail.com \\ Autora: Aguaiza-Tenelema Jessenia Maritza \\ Universidad Laica Eloy Alfaro de Manabí (ULEAM). Manta, Ecuador. \\ jesseniaaguaiza19@hotmail.com
}

\begin{abstract}
RESUMEN
Hoy por hoy, el proceso de comercio no se limita únicamente al periodo de venta directa entre las empresas y consumidor, si no que este se expande hacia nuevos horizontes a través del proceso de postventa. En este marco contextual, las pequeñas y medianas empresas (PYMES) pueden utilizar el modelo de postventa como parte de su metodología estratégica para generar dependencia y preferencias en los consumidores hacia una marca, producto o servicio determinado a través del ofrecimiento de un servicio posterior a la venta de calidad. Consecuentemente, la lealtad de marca desemboca en un desarrollo comercial por en beneficio de las PYMES.
\end{abstract}

Palabras claves: PYMES, consumidor, desarrollo comercial, ventas.

\begin{abstract}
Today, the trade process is not only limited to the period of direct sales between companies and consumers, but it expands towards new horizons through the after-sales process. In this contextual framework, small and medium-sized companies (SMEs) can use the after-sales model as part of their strategic methodology to generate dependence and preferences in consumers towards a specific brand, product or service through the offer of an after-sales service. selling quality. Consequently, brand loyalty leads to commercial development for the benefit of SMEs
\end{abstract}

Keywords: SMEs, consumer, business development, sales.

Información del manuscrito:

Fecha de recepción: 10 de mayo de 2019.

Fecha de aceptación: 09 de julio de 2019.

Fecha de publicación: 10 de julio de 2019. 


\section{INTRODUCCIÓN}

En primera instancia, resulta de suma importancia contextualizar las definiciones etimológicas de postventa y desarrollo comercial, para posteriormente agilizar el entendimiento del impacto del proceso de postventa en el marco del desarrollo comercial correspondiente a las pequeñas y medianas empresas (Pymes). en primer lugar, cabe destacar La postventa, se refiere al proceso posterior a la compra de un producto o servicio. Durante este período, las empresas tienen el deber de llevar a cabo una atención al consumidor dirigida a ejecutar estrategias que mejoren la experiencia de este después de una adquisición. Ineludiblemente con el proceso de la postventa se busca mantener un diálogo inmutable e indeleble con el consumidor, acorde con sus preferencias y necesidades. De este modo es como determinadas marcas se mantienen latentes tanto en la mente como en las elecciones de compra de los consumidores. Indiscutiblemente, el objetivo principal de esta tentativa radica en edificar el dinamismo con el consumidor, puesto que la responsabilidad de las empresas no se limita únicamente al proceso de venta. Si puesto que únicamente verdaderamente si se quiere crear una estructura sólida de consumidores recurrentes que aporten estabilidad a los ingresos de las empresas, resulta ineludible atender las necesidades de los consumidores manifestadas por los mismos.

\section{MATERIALES Y MÉTODO}

\subsection{Postventa y desarrollo comercial en las pymes a nivel global.}

La cultura organizacional ha sido un factor determinante en el éxito o fracaso en los procesos comerciales de cualquier organización. Numerosos son los estudios con referente a la cultura organizacional los cuales han evidenciado el efecto que tiene sobre el rendimiento de las actividades de las organizaciones.

En este marco contextual, Devoto, Liliana. (2019) afirma que las pequeñas y medianas empresas (PYMES) no solo deben ser sostenibles, sino que debe generar beneficios para continuar con las actividades operacionales de la misma. En síntesis, además de ser posible, la sustentabilidad resulta absolutamente necesaria. En este marco teórico la intrínseca necesidad radica al poner marcha 
dos pilares fundamentales, en los cuales predomina la Responsabilidad Social Corporativa (RSC). en primera instancia, resulta optimo tener en cuenta las problemáticas preexistentes, así también como depender del grado de desarrollo referente a determinada locación. A partir de la caracterización de las labores de responsabilidad social, y un sistema de vinculación en la sociedad, resulta de vital importancia desarrollar un marco viable y medible para las pequeñas y medianas empresas que desean no hacer de su responsabilidad una actividad humanística convertida enteramente en una estrategia de imagen corporativa. Concretamente, a través del proceso de la postventa las pequeñas y medianas empresas pueden lograr una integración y desarrollo comercial considerablemente benéfico a largo plazo.

Esto lo corroboran autores como González, A. G. (2014) quien afirma que la calidad de servicio y la satisfacción del cliente se han considerado tradicionalmente como las principales vías para incrementar el índice de fidelidad del consumidor. Este resulta ser del mismo modo el caso del servicio postventa en el contexto de automoción, un sector comercial altamente rentable en países de primera línea como en el caso de España. En este sector comercial se dedica una cantidad significativa de recursos a la mejora de la calidad de servicio.

\subsection{Postventa y desarrollo comercial en las pymes desde el contexto latinoamericano.}

Indiscutiblemente la tecnología en la industria ha mejorado los procesos, bienes y experiencia del consumidor. Debido a esto, la importancia del servicio postventa es una tendencia en aumento. Por ello, autores como Aslam, Wajeeha \& Farhat, Kashif. (2019) otorgan principal importancia a identificar el impacto de la entrega de calidad del servicio posventa en la satisfacción general de los clientes en el sector minorista. Además, el impacto de la satisfacción general en el boca a boca y la intención de recompra. En este contexto, la calidad de la entrega del servicio posventa afecta la satisfacción general del consumidor, lo que conduce a la intención de retorno, generando la lealtad de marca. Por ello, las pequeñas y medianas empresas (PYMES) deben implementar estrategias de marketing, puesto que esto les permite diferenciar sus ofertas para desarrollar la 
relación a largo plazo con sus consumidores, desencadenando tendencias de postventa dinámicas donde los clientes están muy involucrados.

Debido a la creciente globalización actual, el desarrollo tecnológico, así también como los cambios sociopolíticos y económicos, las pequeñas y medianas empresas alrededor del mundo se ven obligadas a involucrarse cada vez más en cuestiones de edificar la innovación en sus procesos de producción. Sin embargo, muchas veces se desconoce cuáles son los factores fundamentales que deben modificarse dentro de la estructura de la empresa con el propósito de elevar su competitividad en el mercado. (Ycaza, D. E. M., \& Sánchez-Riofrío, A. M, 2016). En este marco contextual, resulta optimo afirmar que las denominadas Pymes simbolizan a nivel global un aporte significativo para la economía de los países en que se desarrollan, sin embargo, se deben instaurar mecanismos para que las mismas puedan competir en el mercado frente a las grandes empresas, ya que tienen múltiples desventajas frente a empresas multinacionales de renombre

Pese a esto, autores como Guillermo, Vázquez \& Mejía Trejo, Juan \& Núñez-Moreno, Tania. (2016) afirman que las pymes son vulnerables a presentar diversas problemáticas que no les permiten alcanzar un desarrollo económico de manera óptima y acelerada, puesto que tal como indican, para lograrlo se requiere que estas constantemente implementen estrategias claves y exploren sus propias fortalezas dentro de sus estructuras internas, mismas que al ser analizadas, desencadenen en una búsqueda constante de planes estratégicos que ayuden a maximizarlas.

\subsection{Postventa y desarrollo comercial de las pymes en Ecuador.}

Mejía Zambrano, J. P. (2019) considera que la gestión de las relaciones con los consumidores actualmente constituye una herramienta primordial en el contexto de la toma de decisiones y en el desempeño de los objetivos y estrategias empresariales $u$ organizacionales. Indiscutiblemente, Las tecnologías y los sistemas de información facilitan a las empresas mantener la ventaja competitiva en el mercado, y recíprocamente atender las necesidades y requerimiento de los consumidores. Por ello, resulta de vital importancia de los modelos de la gestión de las relaciones entre distribuidores y consumidores que 
han sido eficientes en varias empresas enfocadas en el ámbito de los servicios, de igual, resulta indispensable contextualizar a las PYMES de los sectores enfocados en la venta de servicios, como en el caso oportuno de la climatización y su actual gestión de consumidores. En este marco contextual, la principal problemática radica en la mala administración y manejo de los consumidores, para lo cual resultaría optimo que las pymes adopten modelos destinados a mejorar sus relaciones con los consumidores con variables y dimensiones aplicables en múltiples contextos, donde la gestión de fidelización de clientes y servicios postventa puedan plantearse como las variables influyentes en este tipo de estrategias. En base a este análisis, resulta acertado afirmar que la cantidad de productos y servicios ofertados, así también como el respectivo seguimiento de los consumidores son los factores no aceptables dentro del modelo de mejora relacional, en el cual las pymes deben centrar sus esfuerzos para forjar una efectiva gestión de clientes.

Esto lo corroboran autores como Morocho, F. R. A., \& Armas, C. S. B. (2017) quienes afirman que, en el sector automotor a nivel de Ecuador, se ha mantenido históricamente una participación considerable en la economía del país, esto debido principalmente a los ingresos que genera en todas las operaciones económicas directas e indirectas que involucra. En este marco contextual, los autores afirman que, a partir de la industria, y el sector económico automotriz se han impulsado otras industrias como la metalúrgica, siderúrgica, metalmecánica, petrolera, minera, petroquímica, entre otras industrias derivadas que resultan indispensables para la manufactura y construcción de vehículos automotores. El sector automotor compone a diferentes actores, como en el caso de las empresas autopartistas, y en específico a proveedoras de partes y piezas automotrices. por otra parte, las empresas ensambladoras de vehículos han sido los actores que impusieron en su momento los estándares productivos en la sucesión de producción de este sector. Dado ello, hoy por hoy, existe una ardua competencia entre las empresas pertenecientes a esta industria con respecto obtener la atención del consumidor. En este contexto, la calidad en el servicio propiciado por las empresas es uno de los factores principales que deben ser implementados dentro de cada una de las empresas. En consecuencia, una eficaz y correcta gestión con respecto a la atención al consumidor resulta en la 
mejor estrategia que se puede implementar para lograr una empresa de éxito comercial, y de esa manera poder experimentar un desarrollo en períodos complicados como el panorama actual que atraviesa el sector automotriz del Ecuador en la época contemporánea.

\section{CONCLUSIONES}

Las PYMES en nuestro país se encuentran en particular en la producción de bienes y servicios, siendo la base del desarrollo social del país tanto produciendo, demandando y comprando productos o añadiendo valor agregado, por lo que se constituyen en un actor fundamental en la generación de riqueza y empleo. Las PYMES favorecen de varias formas al dinamismo del mercado interno debido a que son consumidores de materias primas e insumos nacionales, esto hace pensar que, con una mejor organización, y una planificación estratégica adecuada la sostenibilidad y crecimiento de estas las hace más competitivas no solo en el mercado nacional sino también en mercados internacionales. Es por ello importante el servicio postventa implica seguir ofreciendo una buena atención al cliente aun después de la etapa de venta. Esto tiene el propósito de mantener una excelente relación con el cliente y es tan importante como ofrecer buena atención antes y durante la venta. Hoy en día se abren nuevas empresas diariamente, además de que las ya existentes crean nuevos productos que pueden competir directamente con nosotros. Los clientes tienen tantas opciones que, a pesar de que nos eligieron una vez, eso no asegura que se quedarán con nosotros.

\section{REFERENCIAS}

Aslam, Wajeeha \& Farhat, Kashif. (2019). Impact of after-sales service on consumer behavioural intentions. International Journal of Business and Systems Research. 14. 44. 10.1504/IJBSR.2020.104145.

Delgado, D., \& Chávez, G. (2018). Las Pymes en el Ecuador y sus fuentes de financiamiento. Revista Observatorio de la Economía Latinoamericana, 116. 
Devoto, Liliana. (2019). La sustentabilidad en las pymes, ¿es posible? Cuadernos del Centro de Estudios de Diseño y Comunicación. 10.18682/cdc.vi57.1459.

González, A. G. (2014). Efectos de la calidad de servicio y de la satisfacción del cliente sobre la fidelidad a los servicios oficiales de postventa de automoción españoles (Doctoral dissertation, Universidad de Alcalá).

Guillermo, Vázquez \& Mejía Trejo, Juan \& Núñez-Moreno, Tania. (2016). OPERACIONES Y COMPETITIVIDAD EN LAS PYMES DEL SECTOR DEL PLÁSTICO EN LAS PYMES MANUFACTURERAS DE GUADALAJARA.

Marrero, S. M., \& Solari, E. (2015). Factores de las dificultades de financiación de las Pymes en Latinoamérica. Ciencias administrativas, (6), 3-15.

Mejia Zambrano, J. P. (2019). ARTíCULO CIENTÍFICO: Gestión de clientes basado en el modelo CRM para las Pymes del sector de la climatización de la ciudad de Guayaquil.

Morocho, F. R. A., \& Armas, C. S. B. (2017). Calidad en el Servicio: Oportunidad para el Sector Automotor en el Ecuador. INNOVA Research Journal, 2(9), $42-52$.

Pereira Bolaños, Cliden. (2019). Actualidad de la gestión empresarial en las pymes. Apuntes Contables. 39-53. 10.18601/16577175.n24.03.

Ycaza, D. E. M., \& Sánchez-Riofrío, A. M. (2016). Factores de competitividad orientados a la pequeña y mediana empresa (PYME) en Latinoamérica: revisión de la literatura. Revista San Gregorio, 2(15), 104-111. 